ISSN 1855-3966 (printed edn.), ISSN 1855-3974 (electronic edn.)

\author{
ARS MATHEMATICA CONTEMPORANEA 17 (2019) 185-202 \\ https://doi.org/10.26493/1855-3974.1559.390 \\ (Also available at http://amc-journal.eu)
}

\title{
A diagram associated with the subconstituent algebra of a distance-regular graph
}

\author{
Supalak Sumalroj * \\ Department of Mathematics, Naresuan University, Phitsanulok, Thailand
}

Received 20 December 2017, accepted 6 June 2019, published online 18 September 2019

\begin{abstract}
In this paper we consider a distance-regular graph $\Gamma$. Fix a vertex $x$ of $\Gamma$ and consider the corresponding subconstituent algebra $T=T(x)$. The algebra $T$ is the $\mathbb{C}$-algebra generated by the Bose-Mesner algebra $M$ of $\Gamma$ and the dual Bose-Mesner algebra $M^{*}$ of $\Gamma$ with respect to $x$. We consider the subspaces $M, M^{*}, M M^{*}, M^{*} M, M M^{*} M, M^{*} M M^{*}, \ldots$ along with their intersections and sums. In our notation, $M M^{*}$ means $\operatorname{Span}\{R S \mid R \in$ $\left.M, S \in M^{*}\right\}$, and so on. We introduce a diagram that describes how these subspaces are related. We describe in detail that part of the diagram up to $M M^{*}+M^{*} M$. For each subspace $U$ shown in this part of the diagram, we display an orthogonal basis for $U$ along with the dimension of $U$. For an edge $U \subseteq W$ from this part of the diagram, we display an orthogonal basis for the orthogonal complement of $U$ in $W$ along with the dimension of this orthogonal complement.
\end{abstract}

Keywords: Subconstituent algebra, Terwilliger algebra, distance-regular graph.

Math. Subj. Class.: 05E30

\section{Introduction}

In this paper we consider a distance-regular graph $\Gamma$. Fix a vertex $x$ of $\Gamma$ and consider the corresponding subconstituent algebra (or Terwilliger algebra) $T=T(x)$ [32]. The algebra $T$ is the $\mathbb{C}$-algebra generated by the Bose-Mesner algebra $M$ of $\Gamma$ and the dual Bose-Mesner algebra $M^{*}$ of $\Gamma$ with respect to $x$. The algebra $T$ is finite-dimensional and semisimple [32]. So it is natural to study the irreducible $T$-modules. These modules are used in the study of hypercubes [14, 26], dual polar graphs [20, 38], spin models [6, 10],

*The author would like to thank Professor Paul Terwilliger for many valuable ideas and insightful suggestions on my work. This paper was written while the author was an Honorary Fellow at the University of WisconsinMadison supported by the Development and Promotion of Science and Technology Talents (DPST) Project, Thailand.

E-mail address: supalaks@nu.ac.th (Supalak Sumalroj) 
codes [13, 28], the bipartite property [4, 5, 9, 16, 21, 22, 23, 25, 27], the almost-bipartite property [3, 8, 17], the $Q$-polynomial property [5, 7, 11, 12, 18, 19, 27, 35], and the thin property [15, 24, 30, 31, 33, 34, 36, 37].

In this paper we discuss the algebra $T$ using a different approach. We consider the subspaces $M, M^{*}, M M^{*}, M^{*} M, M M^{*} M, M^{*} M M^{*}, \ldots$ along with their intersections and sums; see Figure 1. We describe the diagram of Figure 1 up to $M M^{*}+M^{*} M$. For each subspace $U$ shown in this part of the diagram, we display an orthogonal basis for $U$ along with the dimension of $U$. For an edge $U \subseteq W$ from this part of the diagram, we display an orthogonal basis for the orthogonal complement of $U$ in $W$ along with the dimension of this orthogonal complement. Our main results are summarized in Theorems 6.1 and 6.2. In the last part of the paper we summarize what is known about the part of diagram above $M M^{*}+M^{*} M$, and we give some open problems.

\section{Preliminaries}

In this section we recall some facts about distance-regular graphs. We will use the following notation. Let $X$ denote a nonempty finite set. Let $\operatorname{Mat}_{X}(\mathbb{C})$ denote the $\mathbb{C}$-algebra consisting of the matrices whose rows and columns are indexed by $X$ and whose entries are in $\mathbb{C}$. For $B \in \operatorname{Mat}_{X}(\mathbb{C})$ let $\bar{B}, B^{t}$, and $\operatorname{tr}(B)$ denote the complex conjugate, the transpose, and the trace of $B$, respectively. We endow $\operatorname{Mat}_{X}(\mathbb{C})$ with the Hermitean inner product $\langle$,$\rangle such that \langle R, S\rangle=\operatorname{tr}\left(R^{t} \bar{S}\right)$ for all $R, S \in \operatorname{Mat}_{X}(\mathbb{C})$. The inner product $\langle$,$\rangle is positive definite. Let U, V$ denote subspaces of $\operatorname{Mat}_{X}(\mathbb{C})$ such that $U \subseteq V$. The orthogonal complement of $U$ in $V$ is defined by $U^{\perp}=\{v \in V \mid\langle v, u\rangle=0$ for all $u \in U\}$.

Let $\Gamma=(X, \mathcal{E})$ denote a finite, undirected, connected graph, without loops or multiple edges, with vertex set $X$ and edge set $\mathcal{E}$. Let $\partial$ denote the shortest path-length distance function for $\Gamma$. Define the diameter $D:=\max \{\partial(x, y) \mid x, y \in X\}$. For a vertex $x \in X$ and an integer $i \geq 0$ define $\Gamma_{i}(x)=\{y \in X \mid \partial(x, y)=i\}$. For notational convenience abbreviate $\Gamma(x)=\Gamma_{1}(x)$. For an integer $k \geq 0$, we say that $\Gamma$ is regular with valency $k$ whenever $|\Gamma(x)|=k$ for all $x \in X$. We say that $\Gamma$ is distance-regular whenever for all integers $h, i, j(0 \leq h, i, j \leq D)$ and $x, y \in X$ with $\partial(x, y)=h$, the number

$$
p_{i j}^{h}:=\left|\Gamma_{i}(x) \cap \Gamma_{j}(y)\right|
$$

is independent of $x$ and $y$. The integers $p_{i j}^{h}$ are called the intersection numbers of $\Gamma$. From now on assume that $\Gamma$ is distance-regular with diameter $D \geq 3$. We abbreviate $k_{i}:=p_{i i}^{0}$ $(0 \leq i \leq D)$. For $0 \leq i \leq D$ let $A_{i}$ denote the matrix in $\operatorname{Mat}_{X}(\mathbb{C})$ with $(x, y)$-entry

$$
\left(A_{i}\right)_{x y}=\left\{\begin{array}{ll}
1 & \text { if } \partial(x, y)=i, \\
0 & \text { if } \partial(x, y) \neq i,
\end{array} \quad x, y \in X .\right.
$$

We call $A_{i}$ the $i$-th distance matrix of $\Gamma$. We call $A=A_{1}$ the adjacency matrix of $\Gamma$. Observe that $A_{i}$ is real and symmetric for $0 \leq i \leq D$. Note that $A_{0}=I$ is the identity matrix in $\operatorname{Mat}_{X}(\mathbb{C})$. Observe that $\sum_{i=0}^{D} A_{i}=J$, where $J$ is the all-ones matrix in $\operatorname{Mat}_{X}(\mathbb{C})$. Observe that for $0 \leq i, j \leq D$,

$$
A_{i} A_{j}=\sum_{h=0}^{D} p_{i j}^{h} A_{h}
$$


For integers $h, i, j(0 \leq h, i, j \leq D)$ we have

$$
\begin{aligned}
& p_{0 j}^{h}=\delta_{h j}, \\
& p_{i j}^{0}=\delta_{i j} k_{i} .
\end{aligned}
$$

Let $M$ denote the subalgebra of $\operatorname{Mat}_{X}(\mathbb{C})$ generated by $A$. By [2, p. 44] the matrices $A_{0}, A_{1}, \ldots, A_{D}$ form a basis for $M$. We call $M$ the Bose-Mesner algebra of $\Gamma$. By [1, p. 59, 64], $M$ has a basis $E_{0}, E_{1}, \ldots, E_{D}$ such that

(i) $E_{0}=|X|^{-1} J$;

(ii) $\sum_{i=0}^{D} E_{i}=I$;

(iii) $E_{i}^{t}=E_{i}(0 \leq i \leq D)$;

(iv) $\overline{E_{i}}=E_{i}(0 \leq i \leq D)$;

(v) $E_{i} E_{j}=\delta_{i j} E_{i}(0 \leq i, j \leq D)$.

The matrices $E_{0}, E_{1}, \ldots, E_{D}$ are called the primitive idempotents of $\Gamma$, and $E_{0}$ is called the trivial idempotent. For $0 \leq i \leq D$ let $m_{i}$ denote the rank of $E_{i}$. For $0 \leq i \leq D$ let $\theta_{i}$ denote an eigenvalue of $A$ associated with $E_{i}$. Let $\lambda$ denote an indeterminate. Define polynomials $\left\{u_{i}\right\}_{i=0}^{D}$ in $\mathbb{C}[\lambda]$ by $u_{0}=1, u_{1}=\lambda / k$, and

$$
\lambda u_{i}=c_{i} u_{i-1}+a_{i} u_{i}+b_{i} u_{i+1} \quad(1 \leq i \leq D-1) .
$$

By $[2$, p. 131, 132],

$$
\begin{array}{ll}
A_{j}=k_{j} \sum_{i=0}^{D} u_{j}\left(\theta_{i}\right) E_{i} & (0 \leq j \leq D), \\
E_{j}=|X|^{-1} m_{j} \sum_{i=0}^{D} u_{i}\left(\theta_{j}\right) A_{i} & (0 \leq j \leq D) .
\end{array}
$$

Since $E_{i} E_{j}=\delta_{i j} E_{i}$ and by (2.4) we have $A_{j} E_{i}=k_{j} u_{j}\left(\theta_{i}\right) E_{i}=E_{i} A_{j}(0 \leq i, j \leq D)$. By [1, Theorem 3.5] we have the orthogonality relations

$$
\begin{array}{ll}
\sum_{i=0}^{D} u_{i}\left(\theta_{r}\right) u_{i}\left(\theta_{s}\right) k_{i}=\delta_{r s} m_{r}^{-1}|X| & (0 \leq r, s \leq D), \\
\sum_{r=0}^{D} u_{i}\left(\theta_{r}\right) u_{j}\left(\theta_{r}\right) m_{r}=\delta_{i j} k_{i}^{-1}|X| & (0 \leq i, j \leq D) .
\end{array}
$$

We recall the Krein parameters of $\Gamma$. Let $\circ$ denote the entry-wise multiplication in $\operatorname{Mat}_{X}(\mathbb{C})$. Note that $A_{i} \circ A_{j}=\delta_{i j} A_{i}$ for $0 \leq i, j \leq D$. So $M$ is closed under $\circ$. By [2, p. 48], there exist scalars $q_{i j}^{h} \in \mathbb{C}$ such that

$$
E_{i} \circ E_{j}=|X|^{-1} \sum_{h=0}^{D} q_{i j}^{h} E_{h} \quad(0 \leq i, j \leq D) .
$$


We call the $q_{i j}^{h}$ the Krein parameters of $\Gamma$. By [2, Proposition 4.1.5], these parameters are real and nonnegative for $0 \leq h, i, j \leq D$.

We recall the dual Bose-Mesner algebra of $\Gamma$. Fix a vertex $x \in X$. For $0 \leq i \leq D$ let $E_{i}^{*}=E_{i}^{*}(x)$ denote the diagonal matrix in $\operatorname{Mat}_{X}(\mathbb{C})$ with $(y, y)$-entry

$$
\left(E_{i}^{*}\right)_{y y}=\left\{\begin{array}{ll}
1 & \text { if } \partial(x, y)=i, \\
0 & \text { if } \partial(x, y) \neq i,
\end{array} \quad y \in X\right.
$$

We call $E_{i}^{*}$ the $i$-th dual idempotent of $\Gamma$ with respect to $x$. Observe that

(i) $\sum_{i=0}^{D} E_{i}^{*}=I$;

(ii) $E_{i}^{* t}=E_{i}^{*}(0 \leq i \leq D)$;

(iii) $\overline{E_{i}^{*}}=E_{i}^{*}(0 \leq i \leq D)$;

(iv) $E_{i}^{*} E_{j}^{*}=\delta_{i j} E_{i}^{*}(0 \leq i, j \leq D)$.

By construction $E_{0}^{*}, E_{1}^{*}, \ldots, E_{D}^{*}$ are linearly independent. Let $M^{*}=M^{*}(x)$ denote the subalgebra of $\operatorname{Mat}_{X}(\mathbb{C})$ with basis $E_{0}^{*}, E_{1}^{*}, \ldots, E_{D}^{*}$. We call $M^{*}$ the dual Bose-Mesner algebra of $\Gamma$ with respect to $x$.

We now recall the dual distance matrices of $\Gamma$. For $0 \leq i \leq D$ let $A_{i}^{*}=A_{i}^{*}(x)$ denote the diagonal matrix in $\operatorname{Mat}_{X}(\mathbb{C})$ with $(y, y)$-entry

$$
\left(A_{i}^{*}\right)_{y y}=|X|\left(E_{i}\right)_{x y} \quad y \in X .
$$

We call $A_{i}^{*}$ the dual distance matrix of $\Gamma$ with respect to $x$ and $E_{i}$. By [32, p. 379], the matrices $A_{0}^{*}, A_{1}^{*}, \ldots, A_{D}^{*}$ form a basis for $M^{*}$. Observe that

(i) $A_{0}^{*}=I$;

(ii) $\sum_{i=0}^{D} A_{i}^{*}=|X| E_{0}^{*}$;

(iii) $A_{i}^{* t}=A_{i}^{*}(0 \leq i \leq D)$;

(iv) $\overline{A_{i}^{*}}=A_{i}^{*}(0 \leq i \leq D)$;

(v) $A_{i}^{*} A_{j}^{*}=\sum_{h=0}^{D} q_{i j}^{h} A_{h}^{*}(0 \leq i, j \leq D)$.

From (2.4) and (2.5) we have

$$
\begin{array}{ll}
A_{j}^{*}=m_{j} \sum_{i=0}^{D} u_{i}\left(\theta_{j}\right) E_{i}^{*} & (0 \leq j \leq D), \\
E_{j}^{*}=|X|^{-1} k_{j} \sum_{i=0}^{D} u_{j}\left(\theta_{i}\right) A_{i}^{*} & (0 \leq j \leq D) .
\end{array}
$$




\section{The subconstituent algebra $T$}

In this section we study the subconstituent algebra of a distance-regular graph. For the rest of the paper, fix a distance-regular graph $\Gamma$ and a vertex $x$ of $\Gamma$. Let $T=T(x)$ denote the subalgebra of $\operatorname{Mat}_{X}(\mathbb{C})$ generated by $M, M^{*}$. The algebra $T$ is called the subconstituent algebra (or Terwilliger algebra) [32]. In order to describe $T$, we consider how $M, M^{*}$ are related. We will use the following notation. For any two subspaces $\mathcal{R}, \mathcal{S}$ of $\operatorname{Mat}_{X}(\mathbb{C})$ we define $\mathcal{R} \mathcal{S}=\operatorname{Span}\{R S \mid R \in \mathcal{R}, S \in \mathcal{S}\}$. Consider the subspaces $M, M^{*}, M M^{*}, M^{*} M, M M^{*} M, M^{*} M M^{*}, \ldots$ along with their intersections and sums. To describe the inclusions among the resulting subspaces we draw a diagram; see Figure 1. In this diagram, a line segment that goes upward from $U$ to $W$ means that $W$ contains $U$.

Consider the diagram in Figure 1. For each subspace $U$ shown in the diagram, we seek an orthogonal basis for $U$ and the dimension of $U$. Also, for each edge $U \subseteq W$ shown in the diagram, we seek an orthogonal basis for the orthogonal complement of $U$ in $W$ along with the dimension of this orthogonal complement. We accomplish these goals for that part of the diagram up to $M M^{*}+M^{*} M$. Our main results are summarized in Theorems 6.1 and 6.2. Before we get started, we recall a few inner product formulas.

Lemma 3.1 ([11, Lemma 3.1, Lemma 4.1]). For $0 \leq h, i, j, r, s, t \leq D$,

(i) $\left\langle E_{i}^{*} A_{j} E_{h}^{*}, E_{r}^{*} A_{s} E_{t}^{*}\right\rangle=\delta_{i r} \delta_{j s} \delta_{h t} k_{h} p_{i j}^{h}$,

(ii) $\left\langle E_{i} A_{j}^{*} E_{h}, E_{r} A_{s}^{*} E_{t}\right\rangle=\delta_{i r} \delta_{j s} \delta_{h t} m_{h} q_{i j}^{h}$.

The following result is well-known.

Lemma 3.2 ([32, Lemma 3.2]). For $0 \leq h, i, j \leq D$,

(i) $E_{i}^{*} A_{h} E_{j}^{*}=0$ if and only if $p_{i j}^{h}=0$,

(ii) $E_{i} A_{h}^{*} E_{j}=0$ if and only if $q_{i j}^{h}=0$.

Lemma 3.3 ([29, Lemma 10]). For $0 \leq h, i, j, r, s, t \leq D$,

$$
\left\langle A_{i} E_{j}^{*} A_{h}, A_{r} E_{s}^{*} A_{t}\right\rangle=\sum_{\ell=0}^{D} k_{\ell} p_{i r}^{\ell} p_{j s}^{\ell} p_{h t}^{\ell} .
$$

\section{The subspace $M+M^{*}$}

Our goal in this section is to analyze the inclusion diagram up to $M+M^{*}$. We begin with the trace of elements in $M$ and $M^{*}$.

Lemma 4.1. For $0 \leq i \leq D$,

(i) $\operatorname{tr}\left(A_{i}\right)=\delta_{0 i}|X|$,

(ii) $\operatorname{tr}\left(E_{i}\right)=m_{i}$,

(iii) $\operatorname{tr}\left(E_{i}^{*}\right)=k_{i}$,

(iv) $\operatorname{tr}\left(A_{i}^{*}\right)=\delta_{0 i}|X|$. 


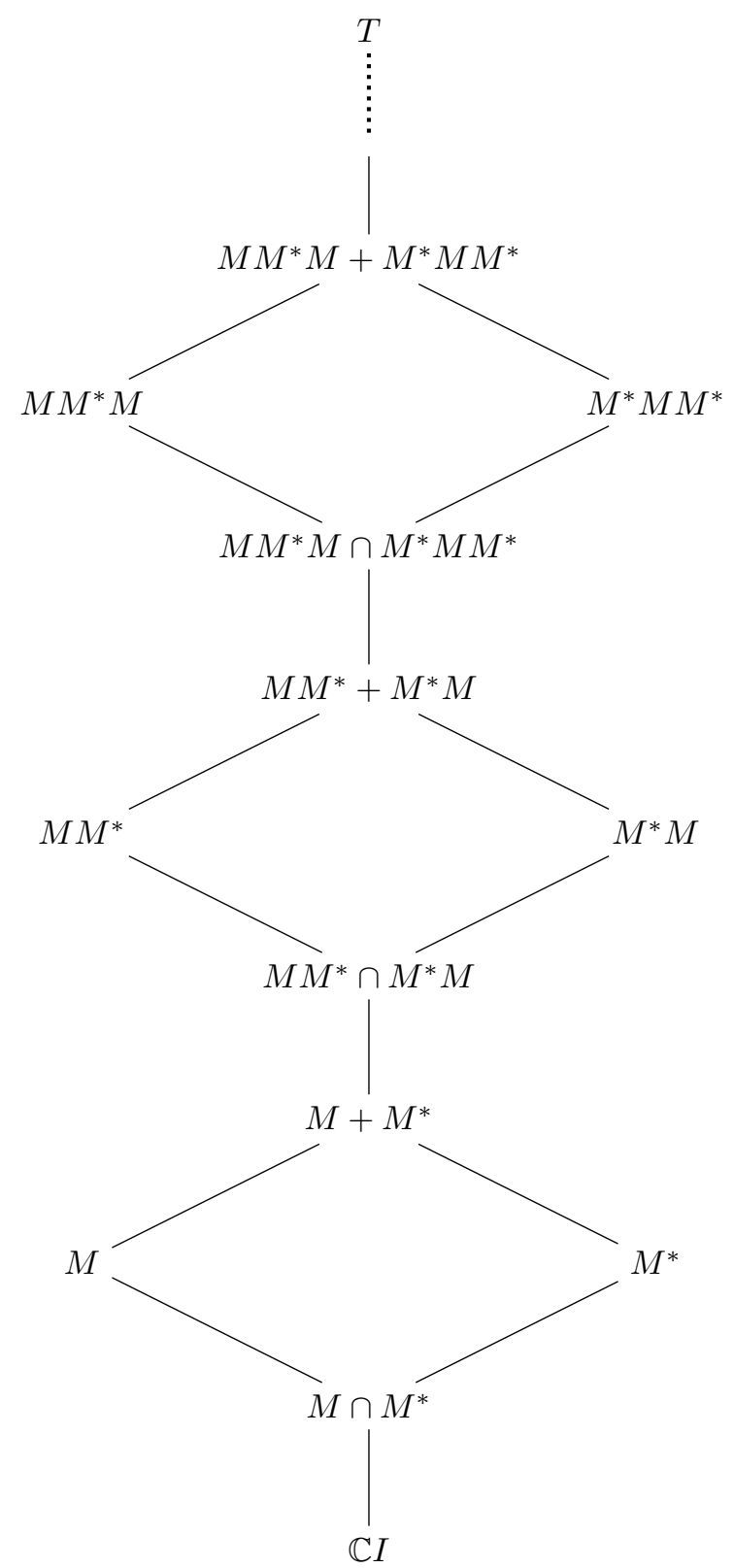

Figure 1: Inclusion diagram. 
Proof. (i): Follows from the definition of $A_{i}$.

(ii): Since $E_{i}$ is diagonalizable, we have $\operatorname{tr}\left(E_{i}\right)=\operatorname{rank}\left(E_{i}\right)=m_{i}$.

(iii): Follows from the definition of $E_{i}^{*}$.

(iv): By (2.5) and since

$$
E_{0}=|X|^{-1} J=|X|^{-1} \sum_{i=0}^{D} A_{i}
$$

we have

$$
\sum_{i=0}^{D}\left(1-u_{i}\left(\theta_{0}\right)\right) A_{i}=0 .
$$

Since $\left\{A_{i}\right\}_{i=0}^{D}$ are linearly independent, we obtain $u_{i}\left(\theta_{0}\right)=1$ for $0 \leq i \leq D$. By (2.6), (2.10) and (iii), we have

$$
\operatorname{tr}\left(A_{i}^{*}\right)=m_{i} \sum_{j=0}^{D} u_{j}\left(\theta_{i}\right) \operatorname{tr}\left(E_{j}^{*}\right)=m_{i} \sum_{j=0}^{D} u_{j}\left(\theta_{i}\right) u_{j}\left(\theta_{0}\right) k_{j}=\delta_{0 i}|X| .
$$

Next we obtain some inner products.

Lemma 4.2. For $0 \leq i, j \leq D$,

(i) $\left\langle A_{i}, A_{j}\right\rangle=\delta_{i j} k_{i}|X|$,

(ii) $\left\langle E_{i}, E_{j}\right\rangle=\delta_{i j} m_{i}$,

(iii) $\left\langle E_{i}^{*}, E_{j}^{*}\right\rangle=\delta_{i j} k_{i}$,

(iv) $\left\langle A_{i}^{*}, A_{j}^{*}\right\rangle=\delta_{i j} m_{i}|X|$.

Proof. (i): Use (2.1) and Lemma 4.1.

(ii): By Lemma 4.1 and since $E_{i} E_{j}=\delta_{i j} E_{i}$.

(iii): Since $E_{i}^{*} E_{j}^{*}=\delta_{i j} E_{i}^{*}(0 \leq i, j \leq D)$ and by Lemma 4.1 (iii).

(iv): By (2.10) and (iii), we obtain

$$
\left\langle A_{i}^{*}, A_{j}^{*}\right\rangle=\left\langle m_{i} \sum_{h=0}^{D} u_{h}\left(\theta_{i}\right) E_{h}^{*}, m_{j} \sum_{\ell=0}^{D} u_{\ell}\left(\theta_{j}\right) E_{\ell}^{*}\right\rangle=m_{i} m_{j} \sum_{h=0}^{D} u_{h}\left(\theta_{i}\right) u_{h}\left(\theta_{j}\right) k_{h} .
$$

By (2.6), we have $\left\langle A_{i}^{*}, A_{j}^{*}\right\rangle=m_{i} m_{j} \delta_{i j} m_{j}^{-1}|X|=\delta_{i j} m_{i}|X|$.

The algebra $M$ has two bases $\left\{A_{i}\right\}_{i=0}^{D}$ and $\left\{E_{i}\right\}_{i=0}^{D}$. The algebra $M^{*}$ has two bases $\left\{A_{i}^{*}\right\}_{i=0}^{D}$ and $\left\{E_{i}^{*}\right\}_{i=0}^{D}$. Next we show that these bases are orthogonal.

Lemma 4.3. Each of the following is an orthogonal basis for $M$ :

$$
\left\{A_{i}\right\}_{i=0}^{D}, \quad\left\{E_{i}\right\}_{i=0}^{D} .
$$

Moreover, each of the following is an orthogonal basis for $M^{*}$ :

$$
\left\{A_{i}^{*}\right\}_{i=0}^{D},
$$$$
\left\{E_{i}^{*}\right\}_{i=0}^{D}
$$ 
Proof. By Lemma 4.2 and the comment below it.

Recall that $A_{0}=I=A_{0}^{*}$. Next we compute some inner products between $M$ and $M^{*}$.

Lemma 4.4. For $0 \leq i, j \leq D$,

$$
\left\langle A_{i}, A_{j}^{*}\right\rangle=\delta_{i 0} \delta_{0 j}|X| k_{i} .
$$

Proof. Observe that $\left\langle A_{i}, A_{j}^{*}\right\rangle=\left\langle A_{i} A_{0}^{*} A_{0}, A_{0} A_{j}^{*} A_{0}\right\rangle$. By Lemma 3.3 and (2.2), (2.6) and (2.10), the result follows.

The next results describe orthogonal bases for $M+M^{*}$ and $M \cap M^{*}$.

Lemma 4.5. The following is an orthogonal basis for $M+M^{*}$ :

$$
A_{D}, \ldots, A_{1}, I, A_{1}^{*} \ldots, A_{D}^{*} .
$$

Proof. Immediate from Lemmas 4.2 and 4.4.

\section{Lemma 4.6.}

$$
\operatorname{dim}\left(M+M^{*}\right)=2 D+1 .
$$

Proof. Immediate from Lemma 4.5.

Lemma 4.7. We have

$$
M \cap M^{*}=\mathbb{C} I \quad \text { and } \quad \operatorname{dim}\left(M \cap M^{*}\right)=1 .
$$

Proof. Observe that $I \in M \cap M^{*}$. By linear algebra, we have

$$
\operatorname{dim}\left(M \cap M^{*}\right)=\operatorname{dim}(M)+\operatorname{dim}\left(M^{*}\right)-\operatorname{dim}\left(M+M^{*}\right) .
$$

By construction $\operatorname{dim}(M)=D+1, \operatorname{dim}\left(M^{*}\right)=D+1$. By this and Lemma 4.6, $\operatorname{dim}\left(M \cap M^{*}\right)=1$. The result follows.

Lemma 4.8. The following statements hold:

(i) The matrices $\left\{A_{i}\right\}_{i=1}^{D}$ form an orthogonal basis for the orthogonal complement of $M \cap M^{*}$ in $M$.

(ii) The matrices $\left\{A_{i}^{*}\right\}_{i=1}^{D}$ form an orthogonal basis for the orthogonal complement of $M \cap M^{*}$ in $M^{*}$.

(iii) The matrices $\left\{A_{i}\right\}_{i=1}^{D}$ form an orthogonal basis for the orthogonal complement of $M^{*}$ in $M+M^{*}$.

(iv) The matrices $\left\{A_{i}^{*}\right\}_{i=1}^{D}$ form an orthogonal basis for the orthogonal complement of $M$ in $M+M^{*}$.

Proof. Follows from definitions of $M, M^{*}$ along with Lemmas 4.5 and 4.7.

Lemma 4.9. Each of the following subspaces has dimension $D$ :

$$
\begin{array}{ll}
\left(M \cap M^{*}\right)^{\perp} \cap M, & \left(M \cap M^{*}\right)^{\perp} \cap M^{*}, \\
\left(M^{*}\right)^{\perp} \cap\left(M+M^{*}\right), & M^{\perp} \cap\left(M+M^{*}\right) .
\end{array}
$$

Proof. Immediate from Lemma 4.8. 


\section{The subspace $M M^{*}+M^{*} M$}

Our goal in this section is to analyze the inclusion diagram from $M+M^{*}$ up to $M M^{*}+M^{*} M$. We begin with a few inner product formulas.

Lemma 5.1. For $0 \leq i, j, r, s \leq D$,

(i) $\left\langle A_{i} A_{j}^{*}, A_{r}^{*} A_{s}\right\rangle=\delta_{i s} \delta_{j r}|X| k_{i} m_{j} u_{i}\left(\theta_{j}\right)$,

(ii) $\left\langle A_{i} A_{j}^{*}, A_{r} A_{s}^{*}\right\rangle=\delta_{i r} \delta_{j s}|X| k_{i} m_{j}$,

(iii) $\left\langle A_{i}^{*} A_{j}, A_{r}^{*} A_{s}\right\rangle=\delta_{i r} \delta_{j s}|X| k_{i} m_{j}$.

Proof. (i): Since

$$
\left\langle A_{i} A_{j}^{*}, A_{r}^{*} A_{s}\right\rangle=\operatorname{tr}\left(A_{j}^{*} A_{i} A_{r}^{*} A_{s}\right)=\sum_{y \in X} \sum_{z \in X}\left(A_{j}^{*}\right)_{y y}\left(A_{i}\right)_{y z}\left(A_{r}^{*}\right)_{z z}\left(A_{s}\right)_{z y}
$$

and by (2.9), it follows that

$$
\begin{aligned}
\left\langle A_{i} A_{j}^{*}, A_{r}^{*} A_{s}\right\rangle & =|X|^{2} \sum_{y \in X} \sum_{z \in X}\left(E_{j}\right)_{x y}\left(A_{i}\right)_{y z}\left(E_{r}\right)_{x z}\left(A_{s}\right)_{z y} \\
& =|X|^{2} \sum_{y \in X} \sum_{z \in X}\left(E_{j}\right)_{x y}\left(A_{i} \circ A_{s}\right)_{y z}\left(E_{r}\right)_{z x} .
\end{aligned}
$$

Since $A_{i} \circ A_{s}=\delta_{i s} A_{i}(0 \leq i, s \leq D)$, we get

$$
\left\langle A_{i} A_{j}^{*}, A_{r}^{*} A_{s}\right\rangle=|X|^{2} \delta_{i s} \sum_{y \in X} \sum_{z \in X}\left(E_{j}\right)_{x y}\left(A_{i}\right)_{y z}\left(E_{r}\right)_{z x} .
$$

Since

$$
\sum_{y \in X} \sum_{z \in X}\left(E_{j}\right)_{x y}\left(A_{i}\right)_{y z}\left(E_{r}\right)_{z x}=|X|^{-1} \operatorname{tr}\left(E_{j} A_{i} E_{r}\right)
$$

we have

$$
\left\langle A_{i} A_{j}^{*}, A_{r}^{*} A_{s}\right\rangle=|X| \delta_{i s} \operatorname{tr}\left(E_{j} A_{i} E_{r}\right)=|X| \delta_{i s} \operatorname{tr}\left(E_{r} E_{j} A_{i}\right) .
$$

Since $E_{i} E_{j}=\delta_{i j} E_{i}(0 \leq i, j \leq D)$, we obtain

$$
\left\langle A_{i} A_{j}^{*}, A_{r}^{*} A_{s}\right\rangle=|X| \delta_{i s} \delta_{j r} \operatorname{tr}\left(E_{j} A_{i}\right)=|X| \delta_{i s} \delta_{j r}\left\langle E_{j}, A_{i}\right\rangle .
$$

By (2.5) and Lemma 4.2 (i), we get $\left\langle E_{j}, A_{i}\right\rangle=m_{j} u_{i}\left(\theta_{j}\right) k_{i}$. Hence

$$
\left\langle A_{i} A_{j}^{*}, A_{r}^{*} A_{s}\right\rangle=\delta_{i s} \delta_{j r}|X| k_{i} m_{j} u_{i}\left(\theta_{j}\right) .
$$

(ii): Since $A_{0}=I$, we get $\left\langle A_{i} A_{j}^{*}, A_{r} A_{s}^{*}\right\rangle=\left\langle A_{i} A_{j}^{*} A_{0}, A_{r} A_{s}^{*} A_{0}\right\rangle$. By (2.10), we obtain

$$
\left\langle A_{i} A_{j}^{*}, A_{r} A_{s}^{*}\right\rangle=m_{j} m_{s} \sum_{h=0}^{D} u_{h}\left(\theta_{j}\right) \sum_{\ell=0}^{D} u_{\ell}\left(\theta_{s}\right)\left\langle A_{i} E_{h}^{*} A_{0}, A_{r} E_{\ell}^{*} A_{0}\right\rangle .
$$

From Lemma 3.3 we have

$$
\left\langle A_{i} E_{h}^{*} A_{0}, A_{r} E_{\ell}^{*} A_{0}\right\rangle=\sum_{t=0}^{D} k_{t} p_{i r}^{t} p_{h \ell}^{t} p_{00}^{t} .
$$


By (2.2) and (2.3), we obtain

$$
\begin{aligned}
\left\langle A_{i} A_{j}^{*}, A_{r} A_{s}^{*}\right\rangle & =m_{j} m_{s} \sum_{h=0}^{D} u_{h}\left(\theta_{j}\right) \sum_{\ell=0}^{D} u_{\ell}\left(\theta_{s}\right) k_{0} p_{i r}^{0} p_{h \ell}^{0} \\
& =\delta_{i r} k_{i} m_{j} m_{s} \sum_{h=0}^{D} u_{h}\left(\theta_{j}\right) u_{h}\left(\theta_{s}\right) k_{h} .
\end{aligned}
$$

By (2.6), we get

$$
\left\langle A_{i} A_{j}^{*}, A_{r} A_{s}^{*}\right\rangle=\delta_{i r} k_{i} m_{j} m_{s} \delta_{j s} m_{s}^{-1}|X|=\delta_{i r} \delta_{j s}|X| k_{i} m_{j} .
$$

(iii): Since

$$
\left\langle A_{i}^{*} A_{j}, A_{r}^{*} A_{s}\right\rangle=\operatorname{tr}\left(\left(A_{i}^{*} A_{j}\right)^{t} \overline{\left(A_{r}^{*} A_{s}\right)}\right)=\operatorname{tr}\left(A_{j} A_{i}^{*} A_{r}^{*} A_{s}\right)=\operatorname{tr}\left(A_{i}^{*} A_{r}^{*} A_{s} A_{j}\right)
$$

and

$$
A_{i}^{*} A_{r}^{*}=\sum_{h=0}^{D} q_{i r}^{h} A_{h}^{*}
$$

and by (2.1), we get

$$
\begin{aligned}
\left\langle A_{i}^{*} A_{j}, A_{r}^{*} A_{s}\right\rangle & =\sum_{h=0}^{D} \sum_{\ell=0}^{D} q_{i r}^{h} p_{j s}^{\ell} \operatorname{tr}\left(A_{h}^{*} A_{\ell}\right)=\sum_{h=0}^{D} \sum_{\ell=0}^{D} q_{i r}^{h} p_{j s}^{\ell} \operatorname{tr}\left(A_{\ell} A_{h}^{*}\right) \\
& =\sum_{h=0}^{D} \sum_{\ell=0}^{D} q_{i r}^{h} p_{j s}^{\ell} \operatorname{tr}\left(A_{\ell}^{t} \overline{A_{h}^{*}}\right)=\sum_{h=0}^{D} \sum_{\ell=0}^{D} q_{i r}^{h} p_{j s}^{\ell}\left\langle A_{\ell}, A_{h}^{*}\right\rangle .
\end{aligned}
$$

From Lemma 4.4, we have

$$
\sum_{h=0}^{D} \sum_{\ell=0}^{D} q_{i r}^{h} p_{j s}^{\ell}\left\langle A_{\ell}, A_{h}^{*}\right\rangle=|X| \sum_{h=0}^{D} \sum_{\ell=0}^{D} q_{i r}^{h} p_{j s}^{\ell} \delta_{\ell 0} \delta_{h 0} k_{\ell}=|X| q_{i r}^{0} p_{j s}^{0} k_{0}=|X| q_{i r}^{0} p_{j s}^{0} .
$$

By (2.3) and since $q_{i r}^{0}=\delta_{i r} m_{i}$, we obtain

$$
\left\langle A_{i}^{*} A_{j}, A_{r}^{*} A_{s}\right\rangle=\delta_{i r} \delta_{j s}|X| k_{j} m_{i} .
$$

Next we obtain orthogonal bases for $M M^{*}$ and $M^{*} M$.

Lemma 5.2. The following statements hold:

(i) The matrices $\left\{A_{i} A_{j}^{*} \mid 0 \leq i, j \leq D\right\}$ form an orthogonal basis for $M M^{*}$.

(ii) The matrices $\left\{A_{j}^{*} A_{i} \mid 0 \leq i, j \leq D\right\}$ form an orthogonal basis for $M^{*} M$.

Proof. Immediate from Lemma 5.1.

Lemma 5.3. Each of the following subspaces has dimension $(D+1)^{2}$ :

$$
M M^{*}, \quad M^{*} M \text {. }
$$


Proof. Immediate from Lemma 5.2.

Our next goal is to obtain an orthogonal basis for $M M^{*}+M^{*} M$.

Lemma 5.4. We have

$$
M M^{*}+M^{*} M=\sum_{i=0}^{D} \sum_{j=0}^{D} \operatorname{Span}\left\{A_{i} A_{j}^{*}, A_{j}^{*} A_{i}\right\} \quad \text { (orthogonal direct sum). }
$$

Proof. Immediate from Lemma 5.1.

Corollary 5.5. We have

$$
\operatorname{dim}\left(M M^{*}+M^{*} M\right)=\sum_{i=0}^{D} \sum_{j=0}^{D} \operatorname{dim}\left(\operatorname{Span}\left\{A_{i} A_{j}^{*}, A_{j}^{*} A_{i}\right\}\right) .
$$

Proof. Immediate from Lemma 5.4.

Definition 5.6. For $0 \leq i, j \leq D$ let $H_{i, j}$ denote the $2 \times 2$ matrix of inner products for $A_{i} A_{j}^{*}, A_{j}^{*} A_{i}$.

Lemma 5.7. For $0 \leq i, j \leq D$,

$$
H_{i, j}=|X| k_{i} m_{j}\left(\begin{array}{cc}
1 & u_{i}\left(\theta_{j}\right) \\
u_{i}\left(\theta_{j}\right) & 1
\end{array}\right) .
$$

Proof. Immediate from Lemma 5.1 and Definition 5.6.

Lemma 5.8. For $0 \leq i, j \leq D$ we have

$$
\operatorname{det}\left(H_{i, j}\right)=|X|^{2} k_{i}^{2} m_{j}^{2}\left(1-\left(u_{i}\left(\theta_{j}\right)\right)^{2}\right) .
$$

Proof. Immediate from Lemma 5.7.

Corollary 5.9. For $0 \leq i, j \leq D$, $\operatorname{det}\left(H_{i, j}\right)=0$ if and only if $u_{i}\left(\theta_{j}\right)= \pm 1$.

Proof. Immediate from Lemma 5.8.

Lemma 5.10. The following elements are orthogonal for $0 \leq i, j \leq D$ :

$$
A_{i} A_{j}^{*}+A_{j}^{*} A_{i}, \quad A_{i} A_{j}^{*}-A_{j}^{*} A_{i} .
$$

Moreover

$$
\begin{gathered}
\left\|A_{i} A_{j}^{*}+A_{j}^{*} A_{i}\right\|^{2}=2|X| k_{i} m_{j}\left(1+u_{i}\left(\theta_{j}\right)\right), \\
\left\|A_{i} A_{j}^{*}-A_{j}^{*} A_{i}\right\|^{2}=2|X| k_{i} m_{j}\left(1-u_{i}\left(\theta_{j}\right)\right) .
\end{gathered}
$$

Proof. Immediate from Lemma 5.7.

Lemma 5.11. The following statements hold for $0 \leq i, j \leq D$ :

(i) Assume $u_{i}\left(\theta_{j}\right)=1$. Then $A_{i} A_{j}^{*}=A_{j}^{*} A_{i}$ and this common value is nonzero. 
(ii) Assume $u_{i}\left(\theta_{j}\right)=-1$. Then $A_{i} A_{j}^{*}=-A_{j}^{*} A_{i}$ and this common value is nonzero.

(iii) Assume $u_{i}\left(\theta_{j}\right) \neq \pm 1$. Then $A_{i} A_{j}^{*}, A_{j}^{*} A_{i}$ are linearly independent.

Proof. (i), (ii): Immediate from Lemma 5.10.

(iii): Immediate from Lemma 5.8.

Lemma 5.12. For $0 \leq i, j \leq D$ we give an orthogonal basis for $\operatorname{Span}\left\{A_{i} A_{j}^{*}, A_{j}^{*} A_{i}\right\}$ in Table 1.

Table 1: An orthogonal basis for $\operatorname{Span}\left\{A_{i} A_{j}^{*}, A_{j}^{*} A_{i}\right\}$.

\begin{tabular}{c|c|c} 
Case & Orthogonal basis & Dimension \\
\hline \hline$u_{i}\left(\theta_{j}\right)= \pm 1$ & $A_{i} A_{j}^{*}$ & 1 \\
$u_{i}\left(\theta_{j}\right) \neq \pm 1$ & $A_{i} A_{j}^{*}+A_{j}^{*} A_{i}, A_{i} A_{j}^{*}-A_{j}^{*} A_{i}$ & 2
\end{tabular}

Proof. Follows from Definition 5.6 and Lemmas 5.7 and 5.11.

Corollary 5.13. The following is an orthogonal basis for $M M^{*}+M^{*} M$ :

$$
\begin{aligned}
\left\{A_{i} A_{j}^{*}+A_{j}^{*} A_{i}, A_{i} A_{j}^{*}-A_{j}^{*} A_{i} \mid 0 \leq i, j \leq D, u_{i}\left(\theta_{j}\right)\right. & \neq \pm 1\} \\
& \cup\left\{A_{i} A_{j}^{*} \mid 0 \leq i, j \leq D, u_{i}\left(\theta_{j}\right)= \pm 1\right\} .
\end{aligned}
$$

Proof. Immediate from Lemmas 5.4 and 5.12.

Our next goal is to find the dimension of $M M^{*}+M^{*} M$.

Definition 5.14. Define an integer $P$ as follows:

$$
P=\left|\left\{(i, j) \mid 1 \leq i, j \leq D, u_{i}\left(\theta_{j}\right)= \pm 1\right\}\right| .
$$

Remark 5.15. Recall that $u_{0}\left(\theta_{j}\right)=1$ and $u_{i}\left(\theta_{0}\right)=1$ for $0 \leq i, j \leq D$. By [2, A.5], the graph $\Gamma$ is primitive if and only if $\Gamma_{i}$ is connected for $1 \leq i \leq D$. From Definition 5.14 and [2, Proposition 4.4.7] we have $P=0$ if and only if $\Gamma$ is primitive.

\section{Lemma 5.16.}

$$
\operatorname{dim}\left(M M^{*}+M^{*} M\right)=2 D^{2}+2 D+1-P .
$$

Proof. Immediate from Corollary 5.13 and Definition 5.14.

Our next goal is to obtain an orthogonal basis for $M M^{*} \cap M^{*} M$.

\section{Lemma 5.17.}

$$
\operatorname{dim}\left(M M^{*} \cap M^{*} M\right)=2 D+1+P .
$$

Proof. By linear algebra, we have

$$
\operatorname{dim}\left(M M^{*} \cap M^{*} M\right)=\operatorname{dim}\left(M M^{*}\right)+\operatorname{dim}\left(M^{*} M\right)-\operatorname{dim}\left(M M^{*}+M^{*} M\right) .
$$

By Lemmas 5.3 and 5.16, the result follows. 
Lemma 5.18. The following is an orthogonal basis for $M M^{*} \cap M^{*} M$ :

$$
\left\{A_{i} A_{j}^{*} \mid 0 \leq i, j \leq D, u_{i}\left(\theta_{j}\right)= \pm 1\right\} .
$$

Proof. Immediate from Lemmas 5.11 and 5.17.

We now have orthogonal bases for $M M^{*}, M^{*} M, M M^{*} \cap M^{*} M$ and $M M^{*}+M^{*} M$. The next results establish an orthogonal basis for certain orthogonal complements along with the dimension for these orthogonal complements.

Lemma 5.19. The matrices $\left\{A_{i} A_{j}^{*} \mid 1 \leq i, j \leq D, u_{i}\left(\theta_{j}\right)= \pm 1\right\}$ form an orthogonal basis for the orthogonal complement of $M+M^{*}$ in $M M^{*} \cap M^{*} M$.

Proof. Follows from Lemmas 4.5 and 5.18.

Lemma 5.20. The following statements hold:

(i) The matrices $\left\{A_{i} A_{j}^{*} \mid 1 \leq i, j \leq D, u_{i}\left(\theta_{j}\right) \neq \pm 1\right\}$ form an orthogonal basis for the orthogonal complement of $M M^{*} \cap M^{*} M$ in $M M^{*}$.

(ii) The matrices $\left\{A_{j}^{*} A_{i} \mid 1 \leq i, j \leq D, u_{i}\left(\theta_{j}\right) \neq \pm 1\right\}$ form an orthogonal basis for the orthogonal complement of $M M^{*} \cap M^{*} M$ in $M^{*} M$.

Proof. Follows from Lemmas 5.2 and 5.18.

Lemma 5.21. The following statements hold:

(i) The matrices $\left\{u_{i}\left(\theta_{j}\right) A_{i} A_{j}^{*}-A_{j}^{*} A_{i} \mid 1 \leq i, j \leq D, u_{i}\left(\theta_{j}\right) \neq \pm 1\right\}$ form an orthogonal basis for the orthogonal complement of $M M^{*}$ in $M M^{*}+M^{*} M$.

(ii) The matrices $\left\{A_{i} A_{j}^{*}-u_{i}\left(\theta_{j}\right) A_{j}^{*} A_{i} \mid 1 \leq i, j \leq D, u_{i}\left(\theta_{j}\right) \neq \pm 1\right\}$ form an orthogonal basis for the orthogonal complement of $M^{*} M$ in $M M^{*}+M^{*} M$.

Proof. (i): By Lemma 5.1, for $0 \leq i, j, r, s \leq D$

$$
\begin{aligned}
& \left\langle A_{r} A_{s}^{*}+A_{s}^{*} A_{r}, u_{i}\left(\theta_{j}\right) A_{i} A_{j}^{*}-A_{j}^{*} A_{i}\right\rangle \\
& =u_{i}\left(\theta_{j}\right)\left\langle A_{r} A_{s}^{*}, A_{i} A_{j}^{*}\right\rangle-\left\langle A_{r} A_{s}^{*}, A_{j}^{*} A_{i}\right\rangle+u_{i}\left(\theta_{j}\right)\left\langle A_{s}^{*} A_{r}, A_{i} A_{j}^{*}\right\rangle-\left\langle A_{s}^{*} A_{r}, A_{j}^{*} A_{i}\right\rangle \\
& =\delta_{i r} \delta_{j s}|X| k_{i} m_{j} u_{i}\left(\theta_{j}\right)-\delta_{i r} \delta_{j s}|X| k_{i} m_{j} u_{i}\left(\theta_{j}\right) \\
& \quad+\delta_{i r} \delta_{j s}|X| k_{i} m_{j}\left(u_{i}\left(\theta_{j}\right)\right)^{2}-\delta_{i r} \delta_{j s}|X| k_{i} m_{j} \\
& =\delta_{i r} \delta_{j s}|X| k_{i} m_{j}\left(\left(u_{i}\left(\theta_{j}\right)\right)^{2}-1\right) .
\end{aligned}
$$

By similar arguments,

$$
\left\langle A_{r} A_{s}^{*}-A_{s}^{*} A_{r}, u_{i}\left(\theta_{j}\right) A_{i} A_{j}^{*}-A_{j}^{*} A_{i}\right\rangle=\delta_{i r} \delta_{j s}|X| k_{i} m_{j}\left(1-\left(u_{i}\left(\theta_{j}\right)\right)^{2}\right)
$$

for $0 \leq i, j, r, s \leq D$. By Lemma 5.1 , for $0 \leq i, j, r, s \leq D$

$$
\begin{aligned}
\left\langle A_{r} A_{s}^{*}, u_{i}\left(\theta_{j}\right) A_{i} A_{j}^{*}-A_{j}^{*} A_{i}\right\rangle & =u_{i}\left(\theta_{j}\right)\left\langle A_{r} A_{s}^{*}, A_{i} A_{j}^{*}\right\rangle-\left\langle A_{r} A_{s}^{*}, A_{j}^{*} A_{i}\right\rangle \\
& =\delta_{i r} \delta_{j s}|X| k_{i} m_{j} u_{i}\left(\theta_{j}\right)-\delta_{i r} \delta_{j s}|X| k_{i} m_{j} u_{i}\left(\theta_{j}\right) \\
& =0 .
\end{aligned}
$$

By Lemma 5.2 and Corollary 5.13, the result follows.

(ii): Similar to the proof of (i). 
Lemma 5.22. The following subspace has dimension $P$ :

$$
\left(M+M^{*}\right)^{\perp} \cap\left(M M^{*} \cap M^{*} M\right) .
$$

Proof. Immediate from Definition 5.14 and Lemma 5.19.

Lemma 5.23. Each of the following subspaces has dimension $D^{2}-P$ :

$$
\begin{array}{ll}
\left(M M^{*} \cap M^{*} M\right)^{\perp} \cap M M^{*}, & \left(M M^{*} \cap M^{*} M\right)^{\perp} \cap M^{*} M, \\
\left(M M^{*}\right)^{\perp} \cap\left(M M^{*}+M^{*} M\right), & \left(M^{*} M\right)^{\perp} \cap\left(M M^{*}+M^{*} M\right) .
\end{array}
$$

Proof. Immediate from Definition 5.14 and Lemmas 5.20 and 5.21.

\section{Summary of main results}

In Sections 4 and 5 we obtained an orthogonal basis and the dimension for each subspace $U$ in the diagram of Figure 1 up to $M M^{*}+M^{*} M$. Also, for each edge $U \subseteq W$ shown in this part of the diagram of Figure 1, we obtained an orthogonal basis for the orthogonal complement of $U$ in $W$ along with the dimension of this orthogonal complement. The results are summarized in this section.

Theorem 6.1. In each row of Table 2 we describe a subspace $U$ in the diagram of Figure 1. We give an orthogonal basis for $U$ along with the dimension of $U$.

Table 2: An orthogonal basis for each subspace $U$ in the diagram of Figure 1 along with its dimension.

\begin{tabular}{c|c|c} 
Subspace $U$ & Orthogonal basis for $U$ & Dimension of $U$ \\
\hline \hline$M \cap M^{*}$ & $I$ & 1 \\
$M$ & $\left\{A_{i}\right\}_{i=0}^{D}$ & $D+1$ \\
$M^{*}$ & $\left\{A_{i}^{*}\right\}_{i=0}^{D}$ & $D+1$ \\
$M+M^{*}$ & $\left\{A_{D}, \ldots, A_{1}, I, A_{1}^{*}, \ldots, A_{D}^{*}\right\}$ & $2 D+1$ \\
$M M^{*} \cap M^{*} M$ & $\left\{A_{i} A_{j}^{*} \mid 0 \leq i, j \leq D, u_{i}\left(\theta_{j}\right)= \pm 1\right\}$ & $2 D+1+P$ \\
$M M^{*}$ & $\left\{A_{i} A_{j}^{*} \mid 0 \leq i, j \leq D\right\}$ & $(D+1)^{2}$ \\
$M^{*} M$ & $\left\{A_{j}^{*} A_{i} \mid 0 \leq i, j \leq D\right\}$ & $(D+1)^{2}$ \\
& $\left\{A_{i} A_{j}^{*}+A_{j}^{*} A_{i}, A_{i} A_{j}^{*}-A_{j}^{*} A_{i} \mid\right.$ & \\
$\left.0 \leq i, j \leq D, u_{i}\left(\theta_{j}\right) \neq \pm 1\right\}$ & $2 D^{2}+2 D+1-P$ \\
$M M^{*}+M^{*} M$ & $\left(\begin{array}{l}\left.0 \leq A_{j}^{*} \mid 0 \leq i, j \leq D, u_{i}\left(\theta_{j}\right)= \pm 1\right\} \\
\end{array}\right.$ &
\end{tabular}


Theorem 6.2. In each row of Table 3 we describe an edge $U \subseteq W$ from the diagram of Figure 1. We give an orthogonal basis for the orthogonal complement of $U$ in $W$ along with the dimension of this orthogonal complement.

Table 3: An orthogonal basis for the orthogonal complement of $U$ in $W$ in the diagram of Figure 1 along with the dimension of this orthogonal complement.

\begin{tabular}{c|c|c|c}
$U$ & $W$ & $\begin{array}{c}\text { Orthogonal basis } \\
\text { for } U^{\perp} \cap W\end{array}$ & $\begin{array}{c}\text { Dimension } \\
\text { of } U^{\perp} \cap W\end{array}$ \\
\hline \hline$M \cap M^{*}$ & $M$ & $\left\{A_{i}\right\}_{i=1}^{D}$ & $D$ \\
$M \cap M^{*}$ & $M^{*}$ & $\left\{A_{i}^{*}\right\}_{i=1}^{D}$ & $D$ \\
$M$ & $M+M^{*}$ & $\left\{A_{i}^{*}\right\}_{i=1}^{D}$ & $D$ \\
$M^{*}$ & $M+M^{*}$ & $\left\{A_{i}\right\}_{i=1}^{D}$ & $D$ \\
$M+M^{*}$ & $M M^{*} \cap M^{*} M$ & $\left\{A_{i} A_{j}^{*} \mid 1 \leq i, j \leq D, u_{i}\left(\theta_{j}\right)= \pm 1\right\}$ & $P$ \\
$M M^{*} \cap M^{*} M$ & $M^{*}$ & $\left\{A_{i} A_{j}^{*} \mid 1 \leq i, j \leq D, u_{i}\left(\theta_{j}\right) \neq \pm 1\right\}$ & $D^{2}-P$ \\
$M M^{*} \cap M^{*} M$ & $M^{*} M^{*}$ & $\left\{A_{j}^{*} A_{i} \mid 1 \leq i, j \leq D, u_{i}\left(\theta_{j}\right) \neq \pm 1\right\}$ & $D^{2}-P$ \\
$M^{*}$ & $M M^{*}+M^{*} M$ & $\left\{u_{i}\left(\theta_{j}\right) A_{i} A_{j}^{*}-A_{j}^{*} A_{i} \mid\right.$ \\
$\left.1 \leq i, j \leq D, u_{i}\left(\theta_{j}\right) \neq \pm 1\right\}$ & $D^{2}-P$ \\
$M^{*} M^{*}$ & $M M^{*}+M^{*} M$ & $\left\{A_{i} A_{j}^{*}-u_{i}\left(\theta_{j}\right) A_{j}^{*} A_{i} \mid\right.$ \\
$\left.1 \leq i, j \leq D, u_{i}\left(\theta_{j}\right) \neq \pm 1\right\}$ & $D^{2}-P$
\end{tabular}

\section{Open problems}

In this section, we give some open problems and suggestions for future research. Earlier in the paper we discussed the diagram of Figure 1. In this discussion we analyzed the diagram up to $M M^{*}+M^{*} M$. The remaining part of the diagram is not completely understood. We mention what is known. By Lemma 3.1 the subspace $M^{*} M M^{*}$ has an orthogonal basis $\left\{E_{i}^{*} A_{j} E_{h}^{*} \mid 0 \leq h, i, j \leq D, p_{i j}^{h} \neq 0\right\}$. Similarly, the subspace $M M^{*} M$ has an orthogonal basis $\left\{E_{i} A_{j}^{*} E_{h} \mid 0 \leq h, i, j \leq D, q_{i j}^{h} \neq 0\right\}$.

Problem 7.1. Find an orthogonal basis for the following subspaces:

(i) $M M^{*} M \cap M^{*} M M^{*}$,

(ii) $M M^{*} M+M^{*} M M^{*}$.

Problem 7.2. In each row of Table 4 we give an edge $U \subseteq W$ from the diagram of Figure 1 . Find an orthogonal basis for the orthogonal complement of $U$ in $W$ for the following cases. 
Table 4: Subspaces $U$ and $W$ from the diagram of Figure 1.

\begin{tabular}{c|c}
$U$ & $W$ \\
\hline \hline$M M^{*}+M^{*} M$ & $M M^{*} M \cap M^{*} M M^{*}$ \\
$M M^{*} M \cap M^{*} M M^{*}$ & $M M^{*} M$ \\
$M M^{*} M \cap M^{*} M M^{*}$ & $M^{*} M M^{*}$ \\
$M M^{*} M$ & $M M^{*} M+M^{*} M M^{*}$ \\
$M^{*} M M^{*}$ & $M M^{*} M+M^{*} M M^{*}$
\end{tabular}

\section{References}

[1] E. Bannai and T. Ito, Algebraic Combinatorics I: Association Schemes, The Benjamin/Cummings Publishing Company, Menlo Park, California, 1984.

[2] A. E. Brouwer, A. M. Cohen and A. Neumaier, Distance-Regular Graphs, volume 18 of Ergebnisse der Mathematik und ihrer Grenzgebiete, 3. Folge, Springer-Verlag, Berlin, 1989, doi: 10.1007/978-3-642-74341-2.

[3] J. S. Caughman, M. S. MacLean and P. M. Terwilliger, The Terwilliger algebra of an almostbipartite $P$ - and $Q$-polynomial association scheme, Discrete Math 292 (2005), 17-44, doi: 10.1016/j.disc.2004.12.001.

[4] J. S. Caughman, IV, The Terwilliger algebras of bipartite $P$ - and $Q$-polynomial schemes, Discrete Math. 196 (1999), 65-95, doi:10.1016/s0012-365x(98)00196-4.

[5] J. S. Caughman, IV, Bipartite $Q$-polynomial distance-regular graphs, Graphs Combin. 20 (2004), 47-57, doi:10.1007/s00373-003-0538-8.

[6] J. S. Caughman, IV and N. Wolff, The Terwilliger algebra of a distance-regular graph that supports a spin model, J. Algebraic Combin. 21 (2005), 289-310, doi:10.1007/ s10801-005-6913-1.

[7] D. R. Cerzo, Structure of thin irreducible modules of a $Q$-polynomial distance-regular graph, Linear Algebra Appl. 433 (2010), 1573-1613, doi:10.1016/j.laa.2010.06.005.

[8] B. V. C. Collins, The Terwilliger algebra of an almost-bipartite distance-regular graph and its antipodal 2-cover, Discrete Math. 216 (2000), 35-69, doi:10.1016/s0012-365x(99)00296-4.

[9] B. Curtin, The Terwilliger algebra of a 2-homogeneous bipartite distance-regular graph, $J$. Comb. Theory Ser. B 81 (2001), 125-141, doi:10.1006/jctb.2000.2002.

[10] B. Curtin and K. Nomura, Spin models and strongly hyper-self-dual Bose-Mesner algebras, $J$. Algebraic Combin. 13 (2001), 173-186, doi:10.1023/a:1011297515395.

[11] G. A. Dickie and P. M. Terwilliger, A note on thin P-polynomial and dual-thin $Q$ polynomial symmetric association schemes, J. Algebraic Combin. 7 (1998), 5-15, doi:10.1023/ a:1008690026999.

[12] A. L. Gavrilyuk and J. H. Koolen, The Terwilliger polynomial of a $Q$-polynomial distanceregular graph and its application to pseudo-partition graphs, Linear Algebra Appl. 466 (2015), 117-140, doi:10.1016/j.laa.2014.09.048.

[13] D. Gijswijt, A. Schrijver and H. Tanaka, New upper bounds for nonbinary codes based on the Terwilliger algebra and semidefinite programming, J. Comb. Theory Ser. A 113 (2006), 17191731, doi:10.1016/j.jcta.2006.03.010.

[14] J. T. Go, The Terwilliger algebra of the hypercube, European J. Combin. 23 (2002), 399-429, doi:10.1006/eujc.2000.0514. 
[15] J. T. Go and P. Terwilliger, Tight distance-regular graphs and the subconstituent algebra, European J. Combin. 23 (2002), 793-816, doi:10.1006/eujc.2002.0597.

[16] M. S. Lang, Bipartite distance-regular graphs: the $Q$-polynomial property and pseudo primitive idempotents, Discrete Math. 331 (2014), 27-35, doi:10.1016/j.disc.2014.04.025.

[17] M. S. Lang and P. M. Terwilliger, Almost-bipartite distance-regular graphs with the $Q$ polynomial property, European J. Combin. 28 (2007), 258-265, doi:10.1016/j.ejc.2005.07.004.

[18] J.-H. Lee, $Q$-polynomial distance-regular graphs and a double affine Hecke algebra of rank one, Linear Algebra Appl. 439 (2013), 3184-3240, doi:10.1016/j.laa.2013.08.015.

[19] J.-H. Lee, Nonsymmetric Askey-Wilson polynomials and $Q$-polynomial distance-regular graphs, J. Comb. Theory Ser. A 147 (2017), 75-118, doi:10.1016/j.jcta.2016.11.006.

[20] J.-H. Lee and H. Tanaka, Dual polar graphs, a nil-DAHA of rank one, and non-symmetric dual $q$-Krawtchouk polynomials, Sém. Lothar. Combin. 78B (2017), Art. 42, 12.

[21] M. S. MacLean and Š. Miklavič, On bipartite distance-regular graphs with exactly one nonthin $T$-module with endpoint two, European J. Combin. 64 (2017), 125-137, doi:10.1016/j. ejc.2017.04.004.

[22] M. S. MacLean and Š. Miklavič, On bipartite distance-regular graphs with exactly two irreducible T-modules with endpoint two, Linear Algebra Appl. 515 (2017), 275-297, doi: 10.1016/j.laa.2016.11.021.

[23] M. S. MacLean, Š. Miklavič and S. Penjić, On the Terwilliger algebra of bipartite distanceregular graphs with $\Delta_{2}=0$ and $c_{2}=1$, Linear Algebra Appl. 496 (2016), 307-330, doi: 10.1016/j.laa.2016.01.040.

[24] M. S. MacLean and P. Terwilliger, Taut distance-regular graphs and the subconstituent algebra, Discrete Math. 306 (2006), 1694-1721, doi:10.1016/j.disc.2006.03.046.

[25] M. S. MacLean and P. Terwilliger, The subconstituent algebra of a bipartite distance-regular graph; thin modules with endpoint two, Discrete Math. 308 (2008), 1230-1259, doi:10.1016/j. disc.2007.03.071.

[26] Š. Miklavič, Leonard triples and hypercubes, J. Algebraic Combin. 28 (2008), 397-424, doi: 10.1007/s10801-007-0108-x.

[27] Š. Miklavič and P. Terwilliger, Bipartite $Q$-polynomial distance-regular graphs and uniform posets, J. Algebraic Combin. 38 (2013), 225-242, doi:10.1007/s10801-012-0401-1.

[28] A. Schrijver, New code upper bounds from the Terwilliger algebra and semidefinite programming, IEEE Trans. Inform. Theory 51 (2005), 2859-2866, doi:10.1109/tit.2005.851748.

[29] S. Sumalroj, A characterization of $Q$-polynomial distance-regular graphs using the intersection numbers, Graphs Combin. 34 (2018), 863-877, doi:10.1007/s00373-018-1917-5.

[30] H. Suzuki, The geometric girth of a distance-regular graph having certain thin irreducible modules for the Terwilliger algebra, European J. Combin. 27 (2006), 235-254, doi:10.1016/j.ejc. 2004.08.006.

[31] H. Tanaka, New proofs of the Assmus-Mattson theorem based on the Terwilliger algebra, European J. Combin. 30 (2009), 736-746, doi:10.1016/j.ejc.2008.07.018.

[32] P. Terwilliger, The subconstituent algebra of an association scheme, Part I, J. Algebraic Combin. 1 (1992), 363-388, doi:10.1023/a:1022494701663.

[33] P. Terwilliger, The subconstituent algebra of a distance-regular graph; thin modules with endpoint one, Linear Algebra Appl. 356 (2002), 157-187, doi:10.1016/s0024-3795(02)00376-2.

[34] P. Terwilliger, An inequality involving the local eigenvalues of a distance-regular graph, $J$. Algebraic Combin. 19 (2004), 143-172, doi:10.1023/b:jaco.0000023004.62272.8c. 
[35] P. Terwilliger, The displacement and split decompositions for a $Q$-polynomial distance-regular graph, Graphs Combin. 21 (2005), 263-276, doi:10.1007/s00373-004-0594-8.

[36] P. Terwilliger and C.-w. Weng, Distance-regular graphs, pseudo primitive idempotents, and the Terwilliger algebra, European J. Combin. 25 (2004), 287-298, doi:10.1016/s0195-6698(03) 00114-8.

[37] P. Terwilliger and A. Žitnik, Distance-regular graphs of $q$-Racah type and the universal AskeyWilson algebra, J. Comb. Theory Ser. A 125 (2014), 98-112, doi:10.1016/j.jcta.2014.03.001.

[38] C. Worawannotai, Dual polar graphs, the quantum algebra $U_{q}\left(\mathfrak{s l}_{2}\right)$, and Leonard systems of dual $q$-Krawtchouk type, Linear Algebra Appl. 438 (2013), 443-497, doi:10.1016/j.laa.2012. 08.016. 